ESAIM: PROCEEDINGS, April 2012, Vol. 36, p. 68-72

Danièle Fournier-Prunaret, Laura Gardini, \& Ludwig Reich, Editors

\title{
ON EVOLUTION OF SMALL SPHERES IN THE PHASE SPACE OF A DYNAMICAL SYSTEM*
}

\author{
Boris Gurevich ${ }^{1}$ And Sergei Komech ${ }^{2}$
}

\begin{abstract}
We study the connection between the entropy of a dynamical system and the boundary distortion rate of regions in the phase space of the system.
\end{abstract}

AMS (2000) subject classification. 54C70, 37D20, 34D08.

Keywords. Kolmogorov entropy, Lyapunov exponents, toral automorphisms, synchronized system.

Résumé. Nous étudions la connexion entre l'entropie d'un système dynamique et le taux de distortion au bord dans l'espace des phases du système.

Mots clefs. Entropie de Kolmogorov, exposants de Lyapunov, automorphismes du tore, système synchronisé.

\section{INTRODUCTION}

Let $X=(X, \rho)$ be a metric space. For every $A \subset X$ and $\varepsilon>0$, we denote the $\varepsilon$-neighborhood of $A$ by $\mathcal{O}_{\varepsilon}(A)$. If $A=\{x\}$ is a single point, then $\mathcal{O}_{\varepsilon}(A)$ is the ball of radius $\varepsilon$ centered at $x$, and we denote it by $B(x, \varepsilon)$. Let $\tau: X \rightarrow X$ be a continuous map and let $h_{\mu}(\tau)$ be the Kolmogorov entropy of $\tau$ with respect to an invariant probability measure $\mu$. The following conjecture is motivated by some remarks in [7]:

If $\tau, \mu$ and a function $\varepsilon \mapsto k(\varepsilon) \in \mathbb{N}$ satisfy some general conditions, then

$$
\lim _{\varepsilon \rightarrow 0} \frac{1}{k(\varepsilon)} \ln \frac{\mu\left(\mathcal{O}_{\varepsilon}\left(\tau^{k(\varepsilon)} B(x, \varepsilon)\right)\right)}{\mu(B(x, \varepsilon))}=h_{\mu}(\tau),
$$

where the convergence holds at least in measure.

In particular, we assume that

$$
\lim _{\varepsilon \rightarrow 0} k(\varepsilon)=\infty, \quad \lim _{\varepsilon \rightarrow 0} k(\varepsilon) / \ln \varepsilon=0 .
$$

(One cannot look forward to reasonable results when $k$ and $\varepsilon$ vary independently.)

It turns out that the conjecture is true at least within two classes of dynamical systems in some sense opposite of each other: symbolic systems (the first rigorous results for them were obtained in [2]) and smooth maps. More precisely, (1) can be proved for so-called synchronized subshifts (and hence for all sofic systems) under natural restrictions on $\mu$, and for Anosov diffeomorphisms with SRB (see, e.g., [4]) measures $\mu$.

* The work is partially supported by RFBR grant 11-01-00485-a.

1 Moscow State University \& Institute for Information Transmission Problems of the Russian Academy of Sciences; email: gurevich@mech.math.msu.su.

${ }^{2}$ Institute for Information Transmission Problems of the Russian Academy of Sciences; email: komech@iitp.ru.

(c) EDP Sciences, SMAI 2012 
We begin with formulating a precise result for synchronized subshifts. Then we will prove the above conjecture for hyperbolic automorphisms of tori to show some basic ideas also employed in a nonlinear case.

\section{Statement of Results}

Let $\mathcal{A}$ be a finite alphabet, $S$ a shift transformation defined on $\mathcal{A}^{\mathbb{Z}}$, and let $X$ be an $S$-invariant compact subset of $\mathcal{A}^{\mathbb{Z}}$. We define a metric $\rho$ on $X$ by

$$
\rho(x, y)=\theta^{n(x, y)}, \quad x, y \in X
$$

where $\theta \in(0,1)$ and

$$
n(x, y)=\min \left\{k \in \mathbb{Z}_{+}: x(-k) \neq y(-k) \text { or } x(k) \neq y(k)\right\}
$$

if $x \neq y$, and $n(x, x)=\infty$.

We recall the definition of a synchronized system (see, e.g., [1]).

Definition 1.1. Let $(X, S)$ be a transitive subshift. A word in the alphabet $\mathcal{A}$ is an $X$-block if it is a subblock in some $x \in X$. The pair $(X, S)$ is a synchronized system if there exists an $X$-block $w$ (a 'magic' word) such that if $u w$ and $w v$ (and hence $u, v$ ) are X-blocks, then $u w v$ is also an X-block.

It is known that the family of synchronized systems contains all subshifts of finite type and, more generally, the sofic systems introduced by Weiss [6].

Theorem 1.2. Let $(X, S)$ be a synchronized system and let $\mu$ be an $S$-invariant ergodic probability measure on $X$. Assume that the function $k: \mathbb{R}^{+} \rightarrow \mathbb{Z}^{+}$satisfies (2) and that there exists a 'magic' word $w$ such that

$$
\mu\left(\left\{y \in X: y_{1} \ldots y_{|w|}=w\right\}\right)>0,
$$

where $|w|$ is the length of the word $w$. Then for all $\theta \in(0,1)$ equation (1) holds with convergence in the sense of $L_{\mu}^{1}$.

This theorem is proved in [8].

We now remind the reader of several well-known facts concerning algebraic automorphisms of tori (see, e.g., $[3,5])$.

Let $\tau$ be a hyperbolic automorphism of the torus $\mathbb{T}^{n}=\mathbb{R}^{n} / \mathbb{Z}^{n}$ and let $\mu$ be the Lebesgue (Haar) measure on $\mathbb{T}^{n}$, which is clearly $\tau$-invariant.

We denote the matrix that induces $\tau$ by $A_{\tau}$. If $\lambda_{1}, \ldots, \lambda_{\max }$ are its eigenvalues, then

$$
h_{\mu}(\tau)=\log \prod_{i:\left|\lambda_{i}\right|>1}\left|\lambda_{i}\right| .
$$

Hyperbolicity of $\tau$ implies that $\left|\lambda_{i}\right| \neq 1$ for all $i$.

Let $H_{s}\left(H_{u}\right)$ be the direct sum of the subspaces in $\mathbb{R}^{n}$ corresponding to $\lambda_{i}$ with $\left|\lambda_{i}\right|<1\left(\left|\lambda_{i}\right|>1\right.$, respectively); $H_{s}$ and $H_{u}$ are invariant under $A_{\tau}, H_{s} \cap H_{u}=\{0\}$, and $\mathbb{R}^{n}=H_{s} \oplus H_{u}$.

We will use the following simple statement: there exist constants $a_{s}>0, a_{u}>0, \lambda_{s}<1$ and $\lambda_{u}>1$ such that for all $y_{1} \in H_{s}, y_{2} \in H_{u}$ and $m \in \mathbb{N}$,

$$
\left\|A_{\tau}^{m} y_{1}\right\| \leq a_{s} \lambda_{s}^{m}\left\|y_{1}\right\|, \quad\left\|A_{\tau}^{-m} y_{2}\right\| \leq a_{u} \lambda_{u}^{-m}\left\|y_{2}\right\|,
$$

where $\|\cdot\|$ denotes the Euclidean norm in $\mathbb{R}^{n}$.

We denote the Lebesgue measures on $H_{s}$ and $H_{u}$ by $\nu_{s}$ and $\nu_{u}$, respectively. Let $\lambda=\prod_{i:\left|\lambda_{i}\right|>1}\left|\lambda_{i}\right|$. For any measurable sets $B_{s} \subset H_{s}$ and $B_{u} \subset H_{u}$,

$$
\nu_{s}\left(A_{\tau} B_{s}\right)=\lambda^{-1} \nu_{s}\left(B_{s}\right), \quad \nu_{u}\left(A_{\tau} B_{u}\right)=\lambda \nu_{u}\left(B_{u}\right) .
$$


Theorem 1.3. If $k(\varepsilon)$ satisfies condition (2) and if $\tau$ and $\mu$ are as above, then for each $x \in \mathbb{T}^{n}$ equation (1) holds.

\section{Proof of Theorem 1.3}

Let

$$
H_{s}(y):=y+H_{s}, \quad H_{u}(y)=y+H_{u}, \quad y \in \mathbb{R}^{n} .
$$

Observe that the sets $H_{s}(y)$ and $H_{u}(y), y \in \mathbb{R}^{n}$, induce two partitions of $\mathbb{R}^{n}$ that are invariant under the action of $A_{\tau}$. We will keep the same notation $\nu_{s}$ and $\nu_{u}$ for the Lebesgue measures on $H_{s}(y)$ and $H_{u}(y)$, respectively.

For a set $D \subset \mathbb{R}^{n}$ we denote its $\varepsilon$-neighbourhood in $\mathbb{R}^{n}$ by $O^{\varepsilon}(D)$. If for some $y \in \mathbb{R}^{n}, D \subset H_{s}(y)$ or $D \subset H_{u}(y)$, we denote the $\varepsilon$-neighbourhood of $D$ in the intrinsic metrics of $H_{s}(y)$ or $H_{u}(y)$ by $O_{s}^{\varepsilon}(D)$ or $O_{u}^{\varepsilon}(D)$, respectively.

Let $G_{s, y}$ and $G_{u, y}$ be measurable subsets of $H_{s}(y)$ and $H_{u}(y)$, respectively. We say the set

$$
G_{s, y} \times G_{u, y}:=\left\{z \in \mathbb{R}^{n}: z=y_{s}+y_{u}-y, \quad y_{s} \in G_{s, y}, \quad y_{u} \in G_{u, y}\right\}
$$

is a parallelogram in $\mathbb{R}^{n}$.

For all $y \in \mathbb{R}^{n}, \delta>0$, we put

$$
P^{\delta}(y)=O_{s}^{\delta}(y) \times O_{u}^{\delta}(y) .
$$

Clearly, for a given $y \in \mathbb{R}^{n}$, there exist constants $C_{1}, C_{2}$ depending only on the angle between $H_{s}$ and $H_{u}$ such that

Upper bound. From (6) we obtain

$$
P^{C_{1} \varepsilon}(y) \subset O^{\varepsilon}(y) \subset P^{C_{2} \varepsilon}(y)
$$

$$
A_{\tau}^{m} O^{\varepsilon}(y) \subset A_{\tau}^{m}\left(P^{C_{2} \varepsilon}(y)\right)=A_{\tau}^{m}\left(O_{s}^{C_{2} \varepsilon}(y) \times O_{u}^{C_{2} \varepsilon}(y)\right), \quad m \in \mathbb{Z}_{+} .
$$

It is easy to see that there exists $\gamma_{1}>0$ depending only on the angle between $H_{s}$ and $H_{u}$ such that

$$
O^{\varepsilon}\left(A_{\tau}^{m} P^{C_{2} \varepsilon}(y)\right) \subset O_{s}^{\gamma_{1} \varepsilon}\left(A_{\tau}^{m} O_{s}^{C_{2} \varepsilon}(y)\right) \times O_{u}^{\gamma_{1} \varepsilon}\left(A_{\tau}^{m} O_{u}^{C_{2} \varepsilon}(y)\right), \quad m \in \mathbb{Z}_{+} .
$$

The first inequality in (4) implies that

$$
\operatorname{diam}\left(A_{\tau}^{m} O_{s}^{C_{2} \varepsilon}(y)\right) \leq 2 a_{s} \lambda_{s}^{m} C_{2} \varepsilon
$$

Since $\lambda_{s}<1$ and $\lim _{\varepsilon \rightarrow 0} k(\varepsilon)=\infty$, for sufficiently small $\varepsilon$ (sufficiently large $k(\varepsilon)$ ) we have

$$
A_{\tau}^{k(\varepsilon)} O_{s}^{C_{2} \varepsilon}(y) \subset O_{s}^{\varepsilon}\left(A_{\tau}^{k(\varepsilon)} y\right)
$$

and hence

Therefore

$$
O_{s}^{\gamma_{1} \varepsilon}\left(A_{\tau}^{k(\varepsilon)} O_{s}^{C_{2} \varepsilon}(y)\right) \subset O_{s}^{\varepsilon\left(1+\gamma_{1}\right)}\left(A_{\tau}^{k(\varepsilon)} y\right)
$$

$$
\nu_{s}\left(O_{s}^{\gamma_{1} \varepsilon}\left(A_{\tau}^{k(\varepsilon)} O_{s}^{C_{2} \varepsilon}(y)\right)\right) \leq \nu_{s}\left(O_{s}^{\varepsilon\left(1+\gamma_{1}\right)}\left(A_{\tau}^{k(\varepsilon)} y\right)\right) .
$$

Now we can estimate $\nu_{u}\left(O_{u}^{\gamma_{1} \varepsilon}\left(A_{\tau}^{m} O_{u}^{C_{2} \varepsilon}(y)\right)\right)$ from above. By (4)

$$
d\left(A_{\tau}^{m} y, \partial\left(A_{\tau}^{m} O_{u}^{C_{2} \varepsilon}(y)\right)\right) \geq a_{u}^{-1} \lambda_{u}^{m} C_{2} \varepsilon
$$

where $d$ is the Euclidean metric in $\mathbb{R}^{n}$. Then the set $\Theta\left(A_{\tau}^{k(\varepsilon)} y\right)$ obtained from $A_{\tau}^{k(\varepsilon)} O_{u}^{C_{2} \varepsilon}(y)$ by the homothety with coefficient 2 centered at $A_{\tau}^{k(\varepsilon)} y$ contains, for a sufficiently small $\varepsilon, O_{u}^{\gamma_{1} \varepsilon}\left(A_{\tau}^{k(\varepsilon)} O_{u}^{C_{2} \varepsilon}(y)\right)$. It is evident that

$$
\nu_{u}\left(\Theta\left(A_{\tau}^{k(\varepsilon)} y\right)\right)=2^{n-l} \nu_{u}\left(A_{\tau}^{k(\varepsilon)} O_{u}^{C_{2} \varepsilon}(y)\right),
$$


where $l$ is the dimension of $H_{s}$. Now (5) implies that

$$
\nu_{u}\left(O_{u}^{\gamma_{1} \varepsilon}\left(A_{\tau}^{k(\varepsilon)} O_{u}^{C_{2} \varepsilon}(y)\right)\right) \leq \nu_{u}\left(\Theta\left(A_{\tau}^{k(\varepsilon)} y\right)\right)=2^{n-l} \lambda^{k(\varepsilon)} \nu_{u}\left(O_{u}^{C_{2} \varepsilon}(y)\right) .
$$

Let $\nu$ be the Lebesgue measure on $\mathbb{R}^{n}$. From (8) - (10) we conclude that

$$
\begin{gathered}
\nu\left(O^{\varepsilon}\left(A_{\tau}^{k(\varepsilon)} P^{C_{2} \varepsilon}(y)\right)\right) \leq \\
\leq \gamma_{0} \nu_{s}\left(O_{s}^{\gamma_{1} \varepsilon}\left(A_{\tau}^{k(\varepsilon)} O_{s}^{C_{2} \varepsilon}(y)\right)\right) \nu_{u}\left(O_{u}^{\gamma_{1} \varepsilon}\left(A_{\tau}^{k(\varepsilon)} O_{u}^{C_{2} \varepsilon}(y)\right)\right) \\
\leq \gamma_{0} \alpha_{1}\left(\varepsilon\left(1+\gamma_{1}\right)\right)^{l} 2^{n-l} \lambda^{k(\varepsilon)} \alpha_{2}\left(C_{2} \varepsilon\right)^{n-l}
\end{gathered}
$$

where $\gamma_{0}$ depends only on the angle between $H_{u}$ and $H_{s}$, and $\alpha_{1}, \alpha_{2}$ depend only on $l$.

Lower bound. By (6) and the definition of the parallelogram $P^{C_{1} \varepsilon}(y)$, for each $m \in \mathbb{Z}_{+}$,

$$
O_{s}^{\varepsilon}\left(A_{\tau}^{m} m\right) \times A_{\tau}^{m} O_{u}^{C_{1} \varepsilon}(y) \subset O^{\varepsilon}\left(A_{\tau}^{m} P^{C_{1} \varepsilon}(y)\right) \subset O^{\varepsilon}\left(A_{\tau}^{m} O^{\varepsilon}(y)\right) .
$$

Let $\pi$ denote the natural projection of $\mathbb{R}^{n}$ onto $\mathbb{T}^{n}$. We will use the following lemma to estimate the measure of the set on the left-hand side of (12).

Lemma 2.1. If $\varepsilon$ is small enough, then the restriction of $\pi$ to the set $O^{\varepsilon}\left(A_{\tau}^{k(\varepsilon)} P^{C_{2} \varepsilon}(y)\right)$ is a bijection.

Proof. From the definition of the parallelogram $P^{C_{2} \varepsilon}(y)$ it follows that

$$
\operatorname{diam}\left(P^{C_{2} \varepsilon}(y)\right) \leq 2 C_{2} \varepsilon
$$

Therefore

$$
\operatorname{diam}\left(O^{\varepsilon}\left(A_{\tau}^{k(\varepsilon)} P^{C_{2} \varepsilon}(y)\right)\right) \leq 2 C_{2} \varepsilon\left\|A_{\tau}\right\|^{k(\varepsilon)}+2 \varepsilon .
$$

By $(2) k(\varepsilon)=\mathrm{o}(\ln (\varepsilon))$, hence the right-hand side of (13) tends to 0 as $\varepsilon \rightarrow 0$. But on every set of diameter smaller than 1 the projection $\pi$ is bijective.

The lemma just proved implies that if $\varepsilon$ is small enough, then

$$
\nu\left(O^{\varepsilon}\left(A_{\tau}^{k(\varepsilon)} P^{C_{1} \varepsilon}(y)\right)\right)=\mu\left(\pi\left(O^{\varepsilon}\left(A_{\tau}^{k(\varepsilon)} P^{C_{1} \varepsilon}(y)\right)\right)\right) .
$$

By (5) and (12)

$$
\begin{aligned}
\nu\left(O^{\varepsilon}\left(A_{\tau}^{k(\varepsilon)} P^{C_{1} \varepsilon}(y)\right)\right) & \geq \gamma_{0} \nu_{s}\left(O_{s}^{\varepsilon} A_{\tau}^{k(\varepsilon)}(y)\right) \nu_{u}\left(O_{u}^{C_{1} \varepsilon}(y)\right) \lambda^{k(\varepsilon)} \\
& \geq \gamma_{0} \alpha_{1} \varepsilon^{l} \alpha_{2}\left(C_{1} \varepsilon\right)^{n-l} \lambda^{k(\varepsilon)},
\end{aligned}
$$

where $\gamma_{0}$, as before, depends only on the angle between $H_{s}$ and $H_{u}$.

Given $x \in \mathbb{T}^{n}$ and $y \in \pi^{-1} x$, from (6) and (14) it follows that

$$
\begin{aligned}
\nu\left(O^{\varepsilon}\left(A_{\tau}^{k(\varepsilon)} P^{C_{1} \varepsilon}(y)\right)\right) & \leq \mu\left(O_{\varepsilon}\left(\tau^{k(\varepsilon)} B(x, \varepsilon)\right)\right) \\
& \leq \nu\left(O^{\varepsilon}\left(A_{\tau}^{k(\varepsilon)} P^{C_{2} \varepsilon}(y)\right)\right) .
\end{aligned}
$$

We substitute (11) and (15) into (16) to obtain

$$
\begin{aligned}
\tilde{\gamma} \varepsilon^{n} \lambda^{k(\varepsilon)} C_{1}{ }^{n-l} & \leq \mu\left(O_{\varepsilon}\left(\tau^{k(\varepsilon)} B(x, \varepsilon)\right)\right) \\
& \leq \tilde{\gamma}\left(1+\gamma_{1}\right)^{l} 2^{n-l} \lambda^{k(\varepsilon)} C_{2}{ }^{n-l} \varepsilon^{n}
\end{aligned}
$$


where $\tilde{\gamma}=\gamma_{0} \alpha_{1} \alpha_{2}$.

The measure of the ball $B(x, \varepsilon)$ with a small $\varepsilon$ equals

$$
\mu(B(x, \varepsilon))=\nu\left(O^{\varepsilon}(y)\right)=\alpha_{3} \varepsilon^{n}
$$

where $\alpha_{3}=$ const.

By putting (11), (17), (18) together and taking (3) into account we arrive at (1).

Remark 2.2. For a nonlinear hyperbolic map of a Riemann manifold, the proof inherits these basic constructions. Of course, it is more delicate to estimate the measure of the projections on the stable and unstable manifolds (in general, with nonzero curvature), and we have to use some additional technics to complete the proof. Moreover, we cannot prove (1) for all $x$ and have to content ourself with a weaker kind of convergence.

\section{REFERENCES}

[1] D. Fiebig, U.-R. Fiebig, The authomorphism group of a coded system. Trans. Amer. Math. Soc. 348, 3173-3191, 1996.

[2] B. M. Gurevich, Geometric interpretation of entropy for random processes. AMS Transl. 171, 81-87, 1996.

[3] B. M. Gurevich, Ya. G. Sinai Algebraic automorphisms of the torus and Markov chains, Appendix to the Russian translation of: P. Billingsley, Ergodic theory and information, Wiley, New York, 1965; ( Translation: Ergodicheskaya teoriya i informatsiya, Mir, Moscow, 1969).

[4] B. Hasselblat, A. Katok, A first course in dynamics: with a panorama of recent developments, Camb. Univ. Press, Cambridge, 2003.

[5] P. Walters, An introduction to ergodic theory, Springer-Verlag, New York-Berlin, 1982.

[6] B. Weiss, Subshifts of finite type and sofic systems, Monats. Math. 77, 1973, pp. 462-474.

[7] G. M. Zaslavsky, Chaos in dynamic systems. Harwood, Chur, 1985.

[8] S. Komech, Boundary distortion rate in synchronized systems: Geometrical meaning of entropy, Problems of Information Transmission, Volume 48, Number 1 (March 2012), pp. 11-20. 